\title{
The 1s x-ray absorption pre-edge structures in transition metal oxides
}

\author{
Frank de Groot ${ }^{1}$, György Vankó ${ }^{2}$ and Pieter Glatzel ${ }^{3}$ \\ ${ }^{1}$ Inorganic Chemistry and Catalysis, Utrecht University, Sorbonnelaan 16, 3584 CA Utrecht, \\ Netherlands \\ ${ }^{2}$ KFKI Research Institute for Particle and Nuclear Physics, PO Box 49, H-1525 Budapest, \\ Hungary \\ ${ }^{3}$ European Synchrotron Radiation Facility, BP 220, 38043 Grenoble Cédex, France \\ E-mail: f.m.f.degroot@uu.nl
}

Received 2 October 2008, in final form 18 November 2008

Published 10 February 2009

Online at stacks.iop.org/JPhysCM/21/104207

\begin{abstract}
We develop a general procedure to analyse the pre-edges in 1s x-ray absorption near edge structure (XANES) of transition metal oxides and coordination complexes. Transition metal coordination complexes can be described from a local model with one metal ion. The $1 \mathrm{~s} 3 \mathrm{~d}$ quadrupole transitions are calculated with the charge-transfer multiplet program. Tetrahedral coordination complexes have more intense pre-edge structures due to the local mixing of $3 \mathrm{~d}$ and $4 p$ states, implying a combination of $1 \mathrm{~s} 3 \mathrm{~d}$ quadrupole and $1 \mathrm{~s} 4 \mathrm{p}$ dipole transitions. Divalent transition metal oxides can be described similar to coordination complexes, but for trivalent and tetravalent oxides, additional structures are visible in the pre-edge region due to non-local dipole transitions. The $1 \mathrm{~s} 4 \mathrm{p}$ dipole transitions have large cross section at the $3 \mathrm{~d}$-band region due to the strong metal-metal interactions, which are oxygen mediated. This yields large intensity in the $3 \mathrm{~d}$-band region but at a different energy than the local $1 \mathrm{~s} 3 \mathrm{~d}$ quadrupole transitions because of smaller core-hole effects due to the delocalization of the excited electron.
\end{abstract}

(Some figures in this article are in colour only in the electronic version)

\section{Introduction}

The 1s XANES pre-edge structures of transition metal oxides are reviewed. The general understanding is that the $1 \mathrm{~s}$ core levels can be explained with the single electron excitation model, while the $2 \mathrm{p}$ XAS spectra need a charge-transfer multiplet analysis, in particular for $3 \mathrm{~d}$ transition metal systems. Using the single electron excitation model, the 1s XANES spectra are divided into the main edge due to transitions from the $1 \mathrm{~s}$ core state to the $4 \mathrm{p}$ conduction band. Below the main edge are, in some cases, minor peaks visible that are denoted as pre-edge structure. These pre-edge structures are assigned as quadrupole transitions from the $1 \mathrm{~s}$ core state to the empty $3 \mathrm{~d}$ states. In case the inversion symmetry of the transition metal is broken, the pre-edge gains additional intensity due to the local $3 d 4 p$ wavefunction mixing, effectively allowing dipole transitions to the $4 \mathrm{p}$ character of the $3 \mathrm{~d}$-band.

The dipole selection rules state that the $1 \mathrm{~s}$ core electron is excited to the lowest possible states with $\mathrm{p}$ character. In a firstrow transition metal oxide the lowest empty metal $\mathrm{p}$ state is the $4 \mathrm{p}$-band that is formed from the anti-bonding combinations of the metal $4 p$ states with the oxygen $2 p$ states. We postpone a detailed description of the pre-edge structures and assume for the moment that the pre-edge relates to the quadrupole transitions to the empty $3 \mathrm{~d}$ states.

The theoretical simulation of the transition metal K edge spectra can be performed with any single particle excitation model that describes the empty density of states [1]. One can use band structure codes such as PARATEC [2, 3], WIEN2K [4], CASTEP [5] or real space multiple scattering codes such as FDMNES [6], FEFF [7], CONTINUUM [8] and MXAN [9]. All these codes can reproduce in many cases the metal K edge XANES accurately, where it is noted that because of the 1s lifetime broadening of more than $1 \mathrm{eV}$, these calculations need to be correct only within this resolution.

The first detailed 1s XANES experiments were performed in the early eighties. The interpretation of the spectra was based on calculations either by multiple scattering [10] or band structure methods [11]. Grunes noted that one should include the response to the core hole as well as the excited-electron core-hole interaction, but the good agreement between single particle excitation calculations and experiment 
indicated that such effects could be neglected, at least with the experimental resolution that was available [11]. Detailed band structure calculations of the transition metal $\mathrm{K}$ edges have been performed by Muller et al [12] and early multiple scattering calculations of the transition metal oxide $\mathrm{K}$ edges were carried out by Norman et al [13]. Important additions in these interpretations included the use of extended basis sets and the inclusion of the core-hole potential [14].

More recent band structure and multiple scattering calculations use final-state calculations including the core-hole potential and the $\mathrm{K}$ edge spectrum can be calculated over the whole XANES region. For example, Wu et al analyse the 1s XANES of a series of transition metal oxides (MnO, $\mathrm{FeO}, \mathrm{CoO}, \mathrm{NiO}$ ) and they found a good agreement between their multiple scattering calculations and the experimental spectra [8]. The pre-edge is calculated as a quadrupole transition. In the experimental $\mathrm{K}$ edge spectrum, the preedge is essentially a single peak, which is also found in the calculations with the broadening as applied. Also with multiple scattering calculations using FEFF [7], good agreement between the metal $\mathrm{K}$ edge and the calculated spectrum is found, where it can be shown that the spectral shape essentially reproduces the metal p-projected density of states, for example in the case of $\mathrm{CaO}$ [15]. In these calculations it is important to include the core-hole potential in order to reproduce the details of the spectral shape. Recent band structure calculations are able to reproduce the metal $\mathrm{K}$ edge XANES spectra in great detail. The PARATEC calculations from Cabaret et al [16] reproduce the $\mathrm{TiO}_{2} \mathrm{~K}$ edge including its angular dependence. Juhin et al show the one-to-one correspondence between the empty density of states and the $\mathrm{Cr} \mathrm{K}$ edge XANES of $\mathrm{Cr}$ impurities in $\mathrm{MgAl}_{2} \mathrm{O}_{4}$ [17].

These results show that the 1s XANES can be accurately described with single electron excitation calculations based on multiple scattering or band structure calculations. That such good agreement is found is actually rather surprising, because of the neglect of charge-transfer effects: the 1s core hole will pull down the $3 \mathrm{~d}$ states, yielding, in principle, a combination of a well screened peak and a poorly screened peak, similar to the case of core level photoemission spectra. This phenomenon was originally described by Bair and Goddard [18] and observed by Tolentino et al [19] in case of $\mathrm{La}_{2} \mathrm{CuO}_{4}$. Recently, this model was also used by Collart et al [20], who selectively excite the $1 s^{1} 3 d^{9} \underline{L} 4 p^{1}$ peak and the $1 s^{1} 3 d^{8} 4 p^{1}$ peak in $\mathrm{La}_{2} \mathrm{NiO}_{4}$ to measure the corresponding RXES spectra. As has been discussed, the majority of $\mathrm{K}$ edge XANES simulations omit these charge-transfer effects and still find very good agreement.

Qualitative analysis of transition metal K edges has been important for the practical application of the technique. These qualitative analysis methods are based on the density of states interpretation and use characteristic features of the pre-edge and edge region to determine trends within related compounds. An initial qualitative relation between the 1s XANES spectra with site geometry and valence was provided by Kau et al [21] for copper compounds. In an extensive XANES study on iron minerals, Waychunas found a correlation between the bond angle variance, a measure for the site distortion, and the normalized pre-edge intensity [22]. Wilke and coworkers extended this relation and they determined that there is a correlation between pre-edge intensity with the local site symmetry [23]. In addition they found a correlation between the energy of the pre-edge barycentre correlates with the valence. These rules have been applied to $\mathrm{Fe}$ and $\mathrm{Mn}$ impurities in zeolite systems [24, 25]. Wong et al [26] found that the pre-edge and the edge shift with different energy scales. This provides an internal measure for the valence determination [27]. These empirical correlations show that also without a detailed theoretical foundation, pre-edge and edge structures can be used to derive useful information. The amount of information that can be extracted from an edge analysis can be considerably increased, though, with the development of more detailed theoretical analyses.

In this paper we will refine the interpretation of the $1 \mathrm{~s}$ XANES spectra of transition metal systems. We will show that, in addition to the density of state based structures described above, additional peaks are present depending on the valence, local symmetry and the metal-metal distances.

\section{HERFD-XANES spectra}

An important improvement in the determination of spectral details for the $\mathrm{K}$ pre-edge structure is the development of high-energy resolution fluorescence detected (HERFD) $\mathrm{x}$ ray absorption. XANES spectra can be measured with a fluorescence detector with an energy resolution that is similar to the core hole lifetime broadening. In the energy range of the transition metal $\mathrm{K}$ edges these detectors are based on perfect crystal Bragg optics. The common Rowland geometry uses spherically bent crystals with a suitable Bragg reflection at a specific $\mathrm{x}$-ray emission energy and the $\mathrm{x}$-rays are focussed on a photon counter. These detectors and the experiments that can be performed with them are discussed, for example in the review paper by Glatzel and Bergmann [28] and also in the book Core Level Spectroscopy of Solids [29]. Hamalainen et al [30] showed that in such measurements the life time broadening of the 1s core hole no longer appears in the spectra.

As an example, the cobalt $\mathrm{K}$ edge $\mathrm{X}$-ray absorption spectra of $\mathrm{CoO}$ is given in figure 1 . The normal $\mathrm{K}$ edge XANES spectrum (dashed) is given in comparison with the HERFDXANES (red, solid). The pre-edge is visible between 7706 and $7710 \mathrm{eV}$. The edge start at approximately $7715 \mathrm{eV}$. The large amount of additionally resolved structures in the 1s HERFDXANES spectrum of $\mathrm{CoO}$ can be used as an example to show how accurate the band structure and multiple scattering calculations are in reproducing these features.

\section{The 1s pre-edge structures}

\subsection{The pre-edge structures in coordination complexes}

For the interpretation of the XANES spectra, coordination complexes are considered as isolated metal ions, surrounded by ligands. The general model to interpret pre-edges is to consider them as quadrupole transitions into the empty $3 \mathrm{~d}$ 


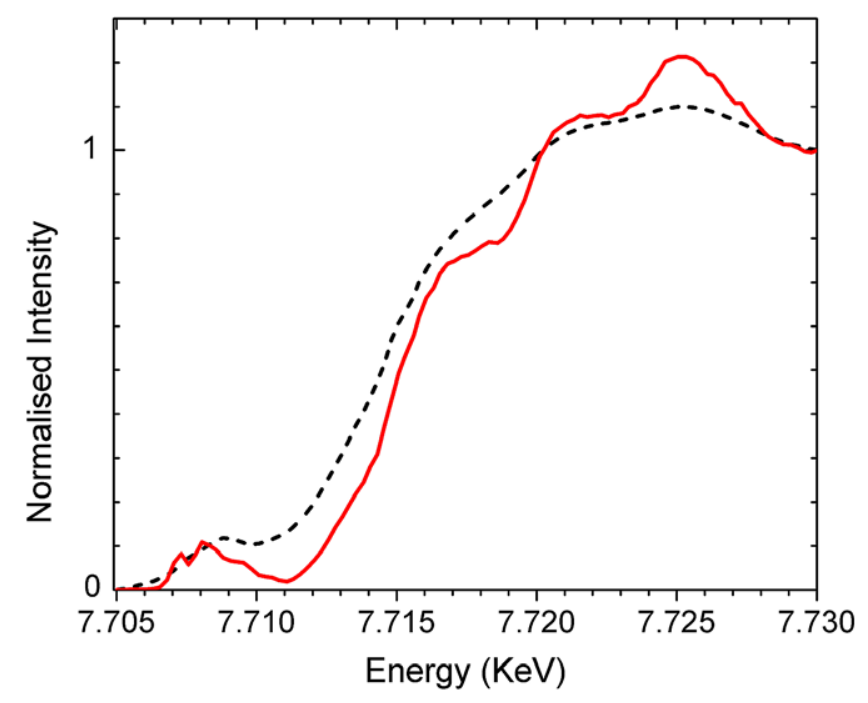

Figure 1. The cobalt 1s XANES spectrum of CoO. Shown are the normal XAS spectrum (dashed), as measured with a solid state fluorescence detector and the HERFD-XANES spectrum (red, solid).

states. Quadrupole transitions have intrinsic transition strength of approximately $0.1 \%$ of the dipole transitions, but because of the high density of states for the 3d-band they are visible as small peaks with $\sim 1-3 \%$ intensity in most, octahedral, transition metal systems. If inversion symmetry is broken, for example in tetrahedral coordination complexes, the metal $3 \mathrm{~d}$ and $4 p$ states mix and there are direct dipole transitions into the $4 \mathrm{p}$ character of the $3 \mathrm{~d}$-band.

Octahedral coordination complexes only have quadrupole transitions and these transitions can be simulated from $3 \mathrm{~d}^{N}$ to $1 \mathrm{~s}^{1} 3 \mathrm{~d}^{N+1}$ multiplet calculations. Figure 2 shows these $3 \mathrm{~d}^{N} \rightarrow 1 \mathrm{~s}^{1} 3 \mathrm{~d}^{N+1}$ calculations, for $N=0-9$. The final state contains a $1 \mathrm{~s}$ core hole, but the $1 \mathrm{~s} 3 \mathrm{~d}$ interaction has no effect on the energy splittings of the pre-edge structures. In fact, the complete structure is determined by the $3 \mathrm{~d}^{N+1}$ multiplet positions and their coupling to the ground state. If one neglects the small $3 \mathrm{~d}$ spin-orbit coupling this approach has been used by Westre et al [31] to explain the pre-edge structures in iron coordination complexes.

It can also be shown that charge-transfer effects have little effect on the 1s $3 \mathrm{~d}$ quadrupole pre-edge spectral shapes. Figure 3 shows the difference between an ionic and a chargetransfer calculation. With the parameters used a significant charge-transfer state was visible in the iron L edge, but the $\mathrm{K}$ edge shows almost no charge-transfer structures. These crystal field multiplet calculations reproduce the pre-edge structures of a series of iron coordination complexes [31, 32] and manganese coordination complexes [33]. In fact, also many bulk transition metal oxide systems can be reproduced with this procedure [34]. The low intensity peaks in figure 3 between 6 and $10 \mathrm{eV}$ will be undetectable, also because the presence of the main edge at these energies. An important difference between $\mathrm{K}$ pre-edge and $\mathrm{L}$ edge structures is that the $\mathrm{L}$ edges are strongly influenced by the multiplet effects of the $2 p$ core hole, i.e. the $2 p 3 d$ two-electron integrals, that redistribute the states and intensities significantly. The 1 s core

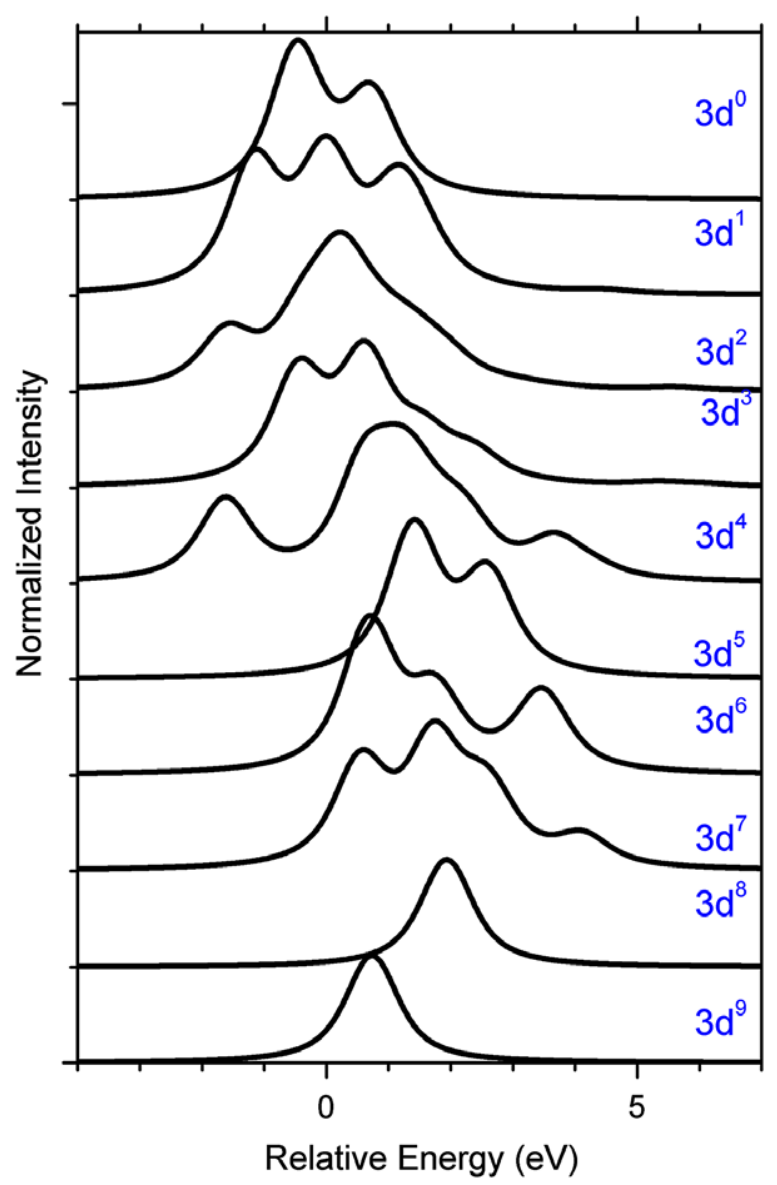

Figure 2. Crystal field multiplet calculations of the $3 \mathrm{~d}^{N}$ to $1 \mathrm{~s}^{1} 3 \mathrm{~d}^{N+1}$ quadrupole transitions with a crystal field value $10 \mathrm{Dq}$ of $1.2 \mathrm{eV}$. The zero energy is set to the average energy of the $3 \mathrm{~d}^{N}$ initial states and the $1 \mathrm{~s}^{1} 3 \mathrm{~d}^{N+1}$ final states.

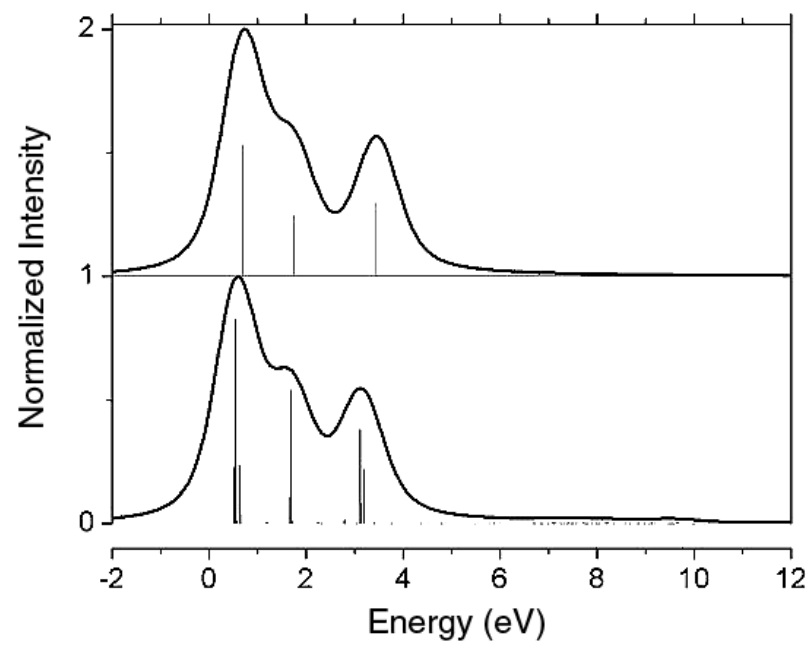

Figure 3. The comparison of a crystal field multiplet calculation (top) with a charge-transfer multiplet calculation (bottom) for a divalent iron coordination complex.

hole has essentially no effect (other than its charge) on the multiplet states, implying that they stay closer to their situation in the ground state. 


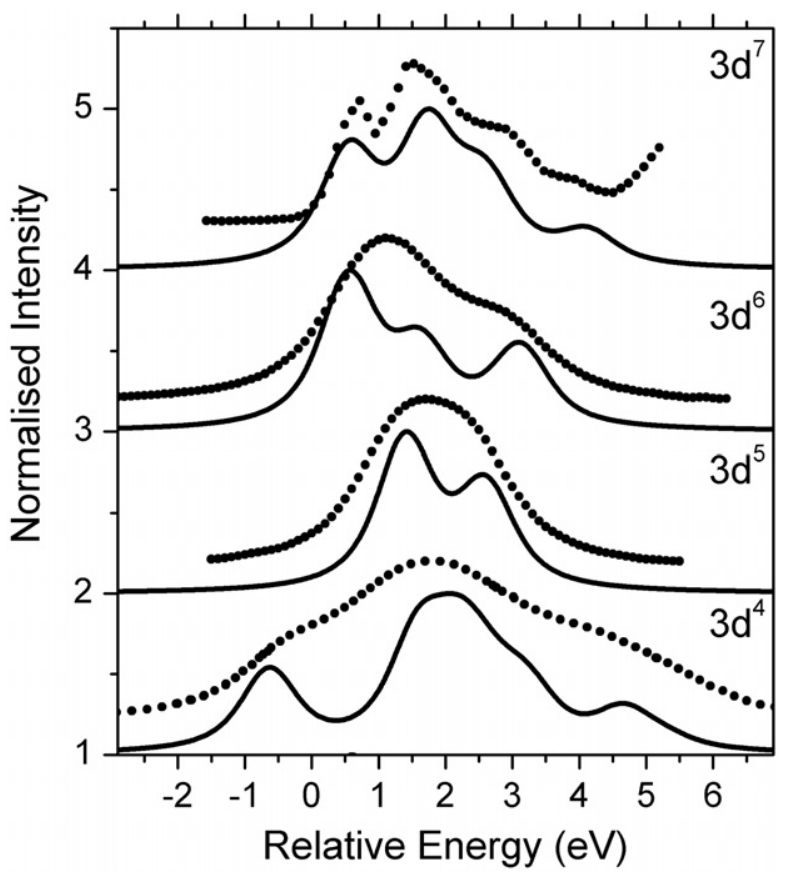

Figure 4. The pre-edge structures of the transition metal oxides $\mathrm{Mn}_{2} \mathrm{O}_{3}\left(3 d^{4}\right), \mathrm{MnO}\left(3 d^{5}\right), \mathrm{Fe}_{2} \mathrm{SiO}_{4}\left(3 d^{6}\right)$ and $\mathrm{CoO}\left(3 d^{7}\right)$ compared with the crystal field multiple calculations as reproduced from figure 2 .

Tetrahedral transition metal coordination complexes, or in general systems where inversion symmetry is broken, have dipole-quadrupole mixing $[31,35]$. In case of tetrahedral symmetry, the local dipole-quadrupole mixing only involves the $t_{2}$-states, not e-states. This has been nicely shown for a series of titanium coordination complexes by de Beer et al [35]. Because inversion symmetry is broken, the metal $3 d$ and $4 p$ states mix with each other and form a set of combined $3 d+4 p$ symmetry states. As such, the dipole (D) and quadrupole (Q) transitions reach the same final states, and their peaks are visible at the same energy.

\subsection{The 1s XANES pre-edge structures in bulk oxides}

The situation of bulk oxides is more complex, because in addition to the local effects, there can be effects due to translation symmetry and/or effects due to metal-metal charge transfer.

Figure 4 compares four experimental pre-edge structures with crystal field multiplet calculations. One can observe that the experimental spectral shapes are relatively well reproduced. The $\mathrm{Mn}_{2} \mathrm{O}_{3}\left(3 \mathrm{~d}^{4}\right)$, $\mathrm{MnO}\left(3 \mathrm{~d}^{5}\right)$ and $\mathrm{Fe}_{2} \mathrm{SiO}_{4}\left(3 \mathrm{~d}^{6}\right)$ have been measured with HERFD-XANES with $1.0 \mathrm{eV}$ overall resolution and $\mathrm{CoO}\left(3 \mathrm{~d}^{7}\right)$ has been measured with $0.3 \mathrm{eV}$ overall resolution. In all cases the constant emission energy (CEE) spectrum is given, in other words the partial fluorescence yield (PFY) spectrum at one particular emission energy. If high resolution is used the PFY spectrum can deviate from the actual $\mathrm{x}$-ray absorption spectrum as will be discussed in section 3.3.

It is difficult to compare these results with the multiple scattering and band structure results as discussed above,

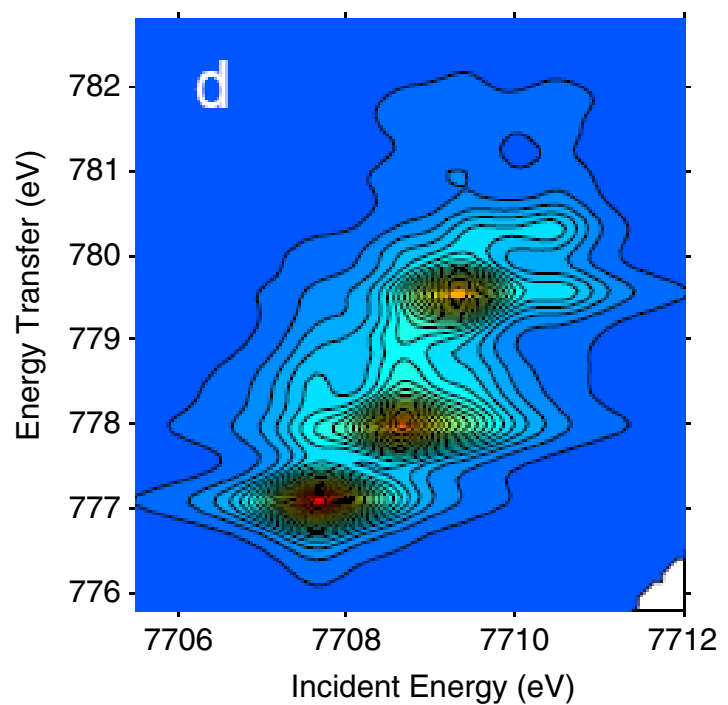

Figure 5. The matrix elements of the $3 d^{7} \rightarrow 1 s^{1} 3 d^{8}$ excitations and the $1 \mathrm{~s}^{1} 3 \mathrm{~d}^{8} \rightarrow 2 \mathrm{p}^{5} 3 \mathrm{~d}^{8}$ decay channels are calculated and inserted into the Kramers-Heisenberg formula.

because in most calculations that analyse the pre-edge structure of normal XANES spectra only a single peak is visible in both experiment and theory [8]. Exceptions are the DFT calculations of $\mathrm{TiO}_{2}$ that reveal two dipole and two quadrupole peaks [16], but for this $3 \mathrm{~d}^{0}$ system there is no difference between multiplet and DFT calculations. Recently, band structure calculations have been used to describe HERFDXANES spectra. The $\mathrm{Fe}_{2} \mathrm{O}_{3}$ spectrum is reproduced by the density of states, as discussed in detail in section 3.4. Again, for this $3 d^{5}$ system, there is no visible difference between multiplet calculations and the DFT results. Juhin et al analysed the angular dependence of the pre-edge structure of the $\mathrm{Cr}$ $\mathrm{K}$ edge of $\mathrm{Cr}^{3+}$ in $\mathrm{MgAl}_{2} \mathrm{O}_{4}$. They show that the angular dependence can only be reproduced with crystal field multiplet calculations, while the DFT calculations do not correctly reproduce this effect [36].

We conclude that for all spectra where the pre-edge structure has been revealed, the crystal field multiplet calculations reproduce the observed spectra. In case of $\mathrm{Cr}^{3+}$, $\mathrm{Fe}^{2+}$ and $\mathrm{Co}^{2+}$ ions in oxides these effects have not (correctly) been calculated by DFT based calculations.

3.2.1. The $1 s$ 2p resonant $x$-ray emission spectrum of $\mathrm{CoO}$. The need for detailed (multiplet) calculations becomes more apparent from the $1 \mathrm{~s} 2 \mathrm{p}$ resonant $\mathrm{x}$-ray emission spectra, or alternatively the resonant inelastic $x$-ray scattering (RIXS). We will show the crystal field multiplet calculations for $\mathrm{CoO}$ as an example. The $1 \mathrm{~s} 3 \mathrm{~d}$ excitation is described as the transition from $3 \mathrm{~d}^{7}$ to $1 \mathrm{~s}^{1} 3 \mathrm{~d}^{8}$ and the $1 \mathrm{~s} 2 \mathrm{p}$ decay is described as the transition from $1 s^{1} 3 d^{8}$ to $2 p^{5} 3 d^{8}$.

Figure 5 shows the $3 \mathrm{~d}^{7} \rightarrow 1 \mathrm{~s}^{1} 3 \mathrm{~d}^{8} \rightarrow 2 \mathrm{p}^{5} 3 \mathrm{~d}^{8}$ transitions in CoO. The Kramers-Heisenberg formula is used and the matrix elements of the $3 \mathrm{~d}^{7} \rightarrow 1 \mathrm{~s}^{1} 3 \mathrm{~d}^{8}$ excitation and the $1 \mathrm{~s}^{1} 3 \mathrm{~d}^{8} \rightarrow 2 \mathrm{p}^{5} 3 \mathrm{~d}^{8}$ decay are included. The spectrum is broadened by the intermediate state $1 \mathrm{~s}$ lifetime broadening, the final-state $2 p$ lifetime broadening plus the 


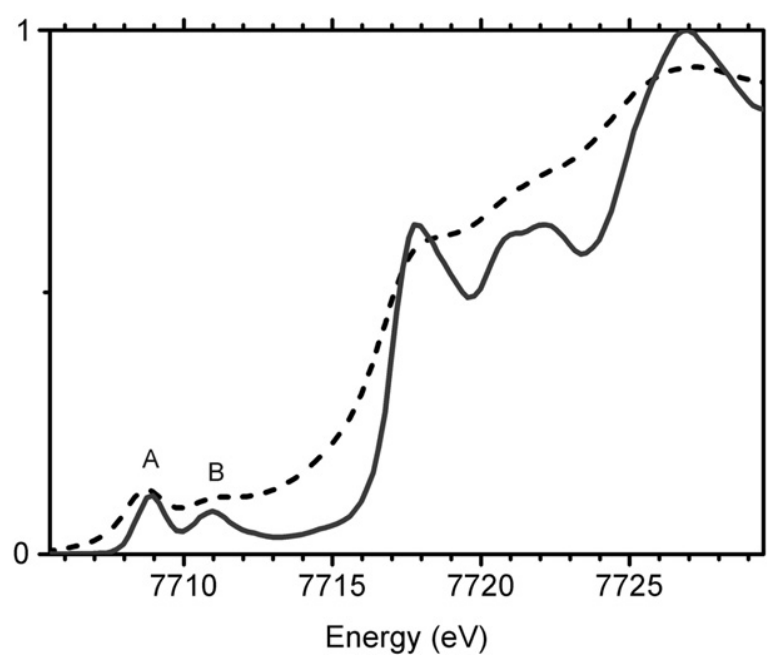

Figure 6. The cobalt 1s XANES spectrum of $\mathrm{LiCoO}_{2}$. Shown is the normal XAS spectrum (dashed), as measured with a solid state fluorescence detector and the HERFD-XANES spectrum (solid).

experimental resolution of the $\mathrm{x}$-ray monochromator and the $\mathrm{x}$-ray detector. The intermediate state $1 \mathrm{~s}$ lifetime broadening plays a role in the denominator of the Kramers-Heisenberg formula and effectively determines the energy range in which states interfere. In case of $\mathrm{CoO}$, the interference effects on the 1s $2 \mathrm{p}$ RIXS spectrum of $\mathrm{CoO}$ are significant due to the large overlap of the $1 \mathrm{~s}^{1} 3 \mathrm{~d}^{8}$ intermediate states [37]. An important result is that the $1 \mathrm{~s} 2 \mathrm{p}$ RIXS spectrum contains many details that can be used for a more precise simulation and hence for a better description of the electronic structure. The recent highresolution experiments agree with these calculations [37]. No additional structure is visible in experiment, other than these, coherent, $3 \mathrm{~d}^{7} \rightarrow 1 \mathrm{~s}^{1} 3 \mathrm{~d}^{8} \rightarrow 2 \mathrm{p}^{5} 3 \mathrm{~d}^{8}$ transitions. For the general interpretation of the pre-edge structures, this implies that the general model of the quadrupole pre-edge plus dipole edge is exactly confirmed in case of $\mathrm{CoO}$.

\subsection{The pre-edge structures in trivalent and tetravalent bulk oxides with inversion symmetry}

Rutile $\mathrm{TiO}_{2}, \mathrm{MnO}_{2}, \quad \mathrm{LiCoO}_{2}$ and $\mathrm{LaCoO}_{3}$ have crystal structures where both the metal-oxygen and the metal-metal bonds have inversion symmetry. We ignore the breaking of inversion symmetry by magnetic ordering for the moment. Inversion symmetry implies the absence of dipole-quadrupole mixing.

Figure 6 shows the cobalt K edge X-ray absorption spectra of $\mathrm{LiCoO}_{2}$. The normal $\mathrm{K}$ edge XANES spectrum is given in comparison with the HERFD-XANES. The peak at $7718 \mathrm{eV}$ is related to the main edge, in other words to the dipole transitions to the $4 \mathrm{p}$-band. The $1 \mathrm{~s}$ XANES spectrum of $\mathrm{LiCoO}_{2}$ contains two clearly observable pre-edges A and B. $\mathrm{LiCoO}_{2}$ is a non-magnetic $(S=0)$ low-spin oxide and its ground state has a filled $\mathrm{t}_{2 \mathrm{~g}}$ band and an empty $\mathrm{e}_{\mathrm{g}}$ band. This implies the presence of a single 1s $3 \mathrm{~d}$ quadrupole peak, which seems in contrast with the two peaks in experiment. Detailed angular dependent experiments showed that the first

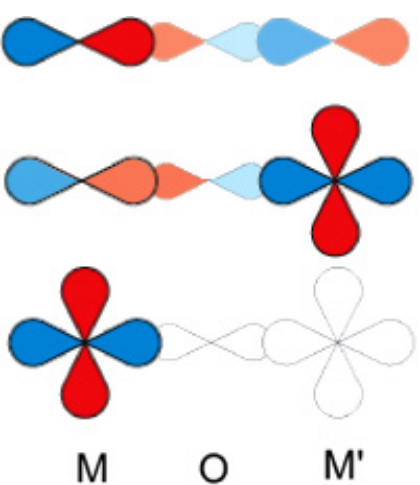

Figure 7. The final-state configurations of the $1 \mathrm{~s} 4 \mathrm{p}$ dipole (top), the non-local $1 \mathrm{~s} 4 \mathrm{p}(3 \mathrm{~d}$ ) dipole (middle) and the $1 \mathrm{~s} 3 \mathrm{~d}$ quadrupole (bottom) transitions.

peak (A) at $7709 \mathrm{eV}$ has quadrupole nature and the second peak (B) at $7711 \mathrm{eV}$ has dipole character [37]. This second preedge peak of dipole character is absent in a Co(aceto-acetate) ${ }_{3}$ coordination complex. Both systems are low-spin trivalent cobalt systems, where $\mathrm{Co}(\mathrm{acac})_{3}$ has isolated $\mathrm{Co}^{\mathrm{III}}$ ions and $\mathrm{LiCoO}_{2}$ contains oxygen mediated $\mathrm{Co}-\mathrm{Co}$ interactions. These observations indicate that the first peak is the local $1 \mathrm{~s} 3 \mathrm{~d}$ quadrupole transition, while the second peak is a dipole $1 \mathrm{~s}$ $4 p$ transition to the $4 p$ character of the $3 d$-band. Because of the inversion symmetry, this $4 p$ character cannot mix with $3 \mathrm{~d}$ character locally, but one can imagine the mixing of $4 p$ character of a neighbouring Co atom into the $3 \mathrm{~d}$ states of the Co atom, where the core hole is excited [37].

Figure 7 indicates the three types of final states, i.e. the $1 \mathrm{~s} 4 \mathrm{p}$ dipole transition to the empty $4 \mathrm{p}$-band (top), the $1 \mathrm{~s} 4 \mathrm{p}$ dipole transition that is coupled to a $3 \mathrm{~d}$ state of a neighbouring Co atom (middle) and the $1 \mathrm{~s} 3 \mathrm{~d}$ quadrupole transition into the local $3 \mathrm{~d}$ states (bottom). Because the core hole is positioned at metal $\mathrm{M}$, the bottom configuration feels a much stronger core-hole potential and as a result it is shifted to lower energy, compared with the middle situation, where the core-hole sites on $\mathrm{M}$ but the $3 \mathrm{~d}$ state is localized at metal $\mathbf{M}^{\prime}$. These nonlocal pre-edge transitions have been observed for the low-spin trivalent cobalt oxides $\mathrm{LiCoO}_{2}, \mathrm{AgCoO}_{2} \mathrm{LaCoO}_{3}$ [37] and also for $\mathrm{TiO}_{2}[16,38,39]$ and $\mathrm{MnO}_{2}$ [40]. Shukla et al observed very small non-local transitions, alternatively called off-site transitions, in $\mathrm{La}_{2} \mathrm{CuO}_{4}$ [41].

\subsection{The pre-edge structures in trivalent bulk oxides without inversion symmetry}

Metal oxides without inversion symmetry allow the local mixing of $4 \mathrm{p}$ and $3 \mathrm{~d}$ states. For example, $\mathrm{Fe}_{2} \mathrm{O}_{3}$ has the corundum structure where the $\mathrm{Fe}-\mathrm{Fe}$ bonds are strongly deviating from inversion symmetry, with essentially aligned short and long $\mathrm{Fe}-\mathrm{Fe}$ bonds. The pre-edge structure of $\mathrm{Fe}_{2} \mathrm{O}_{3}$ shows an angular dependent structure [42] that can be explained as a combination of dipole and quadrupole transitions. Figure 8 compares the pre-edge intensity ratio of the $T_{2 g}$ and $E_{g}$ pre-edge peaks for dipole and quadrupole transitions. The intensity of the dipole transitions has been taken from Glatzel et al [42] and the quadrupole transitions 


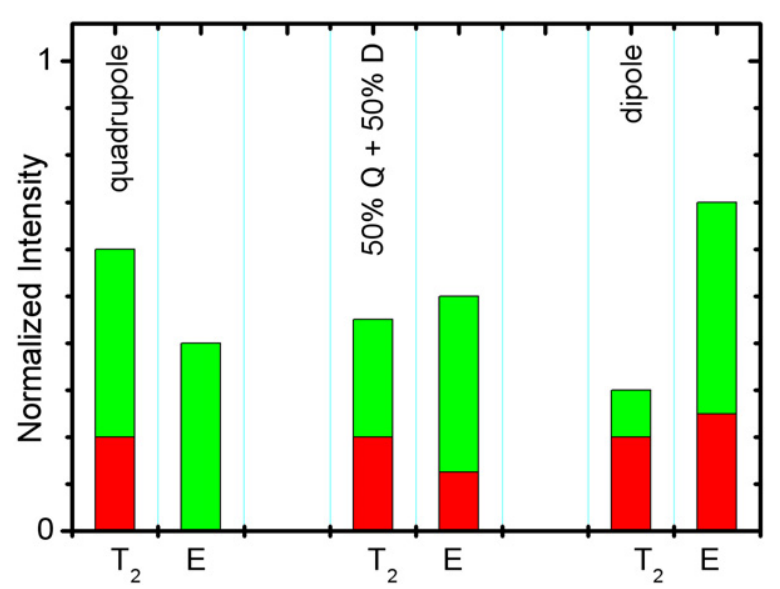

Figure 8. The quadrupole (left) and dipole (right) pre-edge intensities of respectively the $\mathrm{T}_{2 \mathrm{~g}}$ and the $\mathrm{E}_{\mathrm{g}}$ pre-peak in $\mathrm{Fe}_{2} \mathrm{O}_{3}$. The angle-integrated intensities have been divided into intensity for an angle of $45^{\circ}$ with the $c$-axis (red, bottom), respectively perpendicular to the $c$-axis (green, on-top). The middle bars are calculated assuming a 50:50 mixture of dipole and quadrupole transitions.

have been calculated as the $3 \mathrm{~d}^{6} \rightarrow 1 \mathrm{~s}^{1} 3 \mathrm{~d}^{7}$ multiplet transitions in $\mathrm{C}_{3 \mathrm{v}}$ symmetry. The quadrupole transitions have a larger $T_{2 g}$ pre-edge and the dipole transitions a larger $E_{g}$ pre-edge. Both have an angular dependence where $\mathrm{E}_{\mathrm{g}}$ preedge is favoured for the polarization perpendicular to the $c$ axis. The bars in the middle of figure 8 simulate a 50:50 mixture of dipole and quadrupole character, which reproduce the experimental results [42]. This indicates that the pre-edge structures of $\mathrm{Fe}_{2} \mathrm{O}_{3}$ have an approximately $50 \%$ dipole and a $50 \%$ quadrupole character. We note that this is at odds with the results by Dräger et al [43] and further experiments, e.g. angular dependent studies, are necessary. Because of the absence of the inversion symmetry, the $4 p$ states and the $3 \mathrm{~d}$ states can locally mix. The short $\mathrm{Fe}-\mathrm{Fe}$ distance and large distortion from inversion symmetry explain the relatively large dipole component. Also the HERFD-XANES spectra of $\mathrm{Fe}_{2} \mathrm{O}_{3}$ indicate the presence of an additional a pre-edge structure at higher energy, which would be consistent with it being a nonlocal transition similar to the oxides discussed in section 3.3. This interplay between local dipole transitions and non-local dipole transitions remains as yet an open issue that must be further studied [42].

\section{Concluding remarks}

We have given a consistent interpretation of the pre-edge structures in 1s XANES spectra of transition metal oxides and coordination complexes. Transition metal coordination complexes can be well described with a local model containing only one metal ion. Octahedral coordination complexes show weak pre-edge features due to the $1 \mathrm{~s} 3 \mathrm{~d}$ quadrupole transitions and tetrahedral coordination complexes have more intense preedge structures due to the local mixing of $3 \mathrm{~d}$ and $4 \mathrm{~s}$ states. The $1 \mathrm{~s} 3 \mathrm{~d}$ quadrupole transitions are calculated with the chargetransfer multiplet program for the $3 \mathrm{~d}^{N}$ to $1 \mathrm{~s}^{1} 3 \mathrm{~d}^{N+1}$ multiplet transition.
Divalent transition metal oxides can be described similar to coordination coordination complexes, but for trivalent, and tetravalent, oxides, additional structures are visible in the pre-edge region due to non-local dipole transitions, related to oxygen mediated metal-metal interactions. This yields additional intensity at the $3 \mathrm{~d}$-band region, which appears at a different energy than the local 1s $3 \mathrm{~d}$ quadrupole transitions due to the smaller core-hole potential due to the delocalization of the excited electron.

The main edge of the 1s XANES can be accurately described with multiple scattering and band structure calculations. It turns out that in most cases, chargetransfer effects are not detectable and single electron excitation calculations find very good agreement.

\section{Acknowledgments}

Financial support for this research work from the Dutch National Science Foundation (NWO) in the form of a VICI grant (to FMFdG), the Hungarian Scientific Research Fund (OTKA) under contract No. K 72597 (to GV) and the Bolyai János Fellowship (to GV).

\section{References}

[1] Rez P, Bruley J, Brohan P, Payne M and Garvie L A J 1995 Ultramicroscopy 59159

[2] Cabaret D, Mauri F and Henderson G S 2007 Phys. Rev. B 75184205

[3] Gaudry E, Cabaret D, Sainctavit P, Brouder C, Mauri F, Goulon J and Rogalev A 2005 J. Phys.: Condens. Matter 175467

[4] Schwarz K, Blaha P and Madsen G K H 2002 Comput. Phys. Commun. 14771

[5] Milman V, Winkler B, White J A, Pickard C J, Payne M C, Akhmatskaya E V and Nobes R H 2000 Int. J. Quantum Chem. 77895

[6] Joly Y 2003 J. Synchrotron Radiat. 1058

[7] Rehr J J and Albers R C 2000 Rev. Mod. Phys. 72621

[8] Wu Z Y, Xian D C, Hu T D, Xie Y N, Tao Y, Natoli C R, Paris E and Marcelli A 2004 Phys. Rev. B 7033104

[9] Benfatto M, Della Longa S and Natoli C R 2003 J. Synchrotron. Radiat. 1051

[10] Kutzler F W, Scott R A, Berg J M, Hodgson K O, Doniach S, Cramer S P and Chang C H 1981 J. Am. Chem. Soc. 1036083

[11] Grunes L A 1983 Phys. Rev. B 272111

[12] Müller J E, Jepsen O and Wilkins J W 1982 Solid State Commun. 42365

[13] Norman D, Garg K B and Durham P J 1985 Solid State Commun. 56895

[14] Czyzyk M T, Potze R and Sawatzky G A 1992 Phys. Rev. B 463729

[15] Rehr J J and Ankudinov A L 2005 Coord. Chem. Rev. 249131

[16] Cabaret D, Joly Y, Renevier H and Natoli C R 1999 J. Synchrotron. Radiat. 6258

[17] Juhin A, Calas G, Cabaret D, Galoisy L and Hazemann J L 2007 Phys. Rev. B 76054105

[18] Bair R A and Goddard W A III 1980 Phys. Rev. B 222767

[19] Tolentino H, Medarde M, Fontaine A, Baudelet F, Dartyge E, Guay D and Tourillon G 1992 Phys. Rev. B 458091

[20] Collart E, Shukla A, Rueff J P, Leininger P, Ishii H, Jarrige I, Cai Y Q, Cheong S W and Dhalenne G 2006 Phys. Rev. Lett. 96157004 
[21] Kau L S, Spira-Solomon D J, Penner-Hahn J E, Hodgson K O and Solomon E I 1987 J. Am. Chem. Soc. 1096433

[22] Waychunas G A, Apted M J and Brown G E 1983 Phys. Chem. Miner. 101

[23] Wilke M, Farges F, Petit P E, Brown G E and Martin F 2001 Am. Mineral. 86714

[24] Heijboer W M, Glatzel P, Sawant K R, Lobo R F, Bergmann U, Barrea R A, Koningsberger D C, Weckhuysen B M and de Groot F M F 2004 J. Phys. Chem. B 10810002

[25] Radu D, Glatzel P, Gloter A, Stephan O, Weckhuysen B M and de Groot F M F 2008 J. Phys. Chem. C 11212409

[26] Wong J, Lytle F W, Messmer R P and Maylotte D H 1984 Phys. Rev. B 305596

[27] Keller D E, de Groot F M F, Koningsberger D C and Weckhuysen B M 2005 J. Phys. Chem. B 10910223

[28] Glatzel P and Bergmann U 2005 Coord. Chem. Rev. 24965

[29] de Groot F and Kotani A 2008 Core Level Spectroscopy of Solids (New York: Taylor and Francis) p 3

[30] Hämäläinen K, Siddons D P, Hastings J B and Berman L E 1991 Phys. Rev. Lett. 672850

[31] Westre T E, Kennepohl P, DeWitt J G, Hedman B, Hodgson K O and Solomon E I 1997 J. Am. Chem. Soc. 1196297

[32] Vankó G, Neisius T, Molnar G, Renz F, Karpati S, Shukla A and de Groot F M F 2006 J. Phys. Chem. B 11011647
[33] Glatzel P, Bergmann U, Yano J, Visser H, Robblee J H, Gu W W, de Groot F M F, Christou G, Pecoraro V L, Cramer S P and Yachandra V K 2004 J. Am. Chem. Soc. 1269946

[34] de Groot F M F, Glatzel P, Bergmann U, van Aken P A, Barrea R A, Klemme S, Hävecker M, Knop-Gericke A, Heijboer W M and Weckhuysen B M 2005 J. Phys. Chem. B 10920751

[35] DeBeer-George S, Brant P and Solomon E I 2005 J. Am. Chem. Soc. 127667

[36] Juhin A, Brouder C, Arrio M-A, Cabaret D, Sainctavit P, Balan E, Bordage A, Seitsonen A P, Calas G, Eeckhout S G and Glatzel P 2008 Phys. Rev. B 78195103

[37] Vankó G, de Groot F M F, Huotari S, Cava R J, Lorenz T and Reuther M 2008 arXiv:0802.2744

[38] Shirley E L 2004 J. Electron Spectrosc. Relat. Phenom. 13677

[39] Uozumi T, Okada K, Kotani A, Durmeyer O, Kappler J P, Beaurepaire E and Parlebas J C 1992 Europhys. Lett. 1885

[40] Farges F 2005 Phys. Rev. B 71165109

[41] Shukla A, Calandra M, Taguchi M, Kotani A, Vanko G and Cheong S W 2006 Phys. Rev. Lett. 96077006

[42] Glatzel P, Mirone A, Eeckhout S G, Sikora M and Giuli G 2008 Phys. Rev. B 77115133

[43] Dräger G, Frahm R, Materlik G and Brummer O 1988 Phys. Status Solidi b 146287 